\title{
Set-shifting-related basal ganglia deformation as a novel familial marker of obsessive-compulsive disorder
}

Masanori Isobe, Matilde Vaghi, Naomi A. Fineberg, Annemieke M. Apergis-Schoute, Edward T. Bullmore, Barbara J. Sahakian, Trevor W. Robbins and Samuel R. Chamberlain

The symptoms of obsessive-compulsive disorder (OCD) are suggestive of cognitive rigidity, and previous work identified impaired flexible responding on set-shifting tasks in such patients. The basal ganglia are central to habit learning and are thought to be abnormal in OCD, contributing to inflexible, rigid habitual patterns of behaviour. Here, we demonstrate that increased cognitive inflexibility, indexed by poor performance on the set-shifting task, correlated with putamen morphology, and that patients and their asymptomatic relatives had common curvature abnormalities within this same structure. The association between the structure of the putamen and the extradimensional errors was found to be significantly familial in OCD proband-relative pairs. The data implicate changes in basal ganglia structure linked to cognitive inflexibility as a familial marker of OCD. This may reflect a predisposing heightened propensity toward habitual response patterns and deficits in goal-directed planning

\section{Keywords}

Obsessive-compulsive disorder; vulnerability; frontostriatal; basal ganglia; compulsivity.

\section{Copyright and usage}

(C) The Author(s), 2021. Published by Cambridge University Press on behalf of the Royal college of Psychiatrists. This is an Open Access article, distributed under the terms of the Creative Commons Attribution licence (http://creativecommons.org/ licenses/by/4.0/), which permits unrestricted re-use, distribution, and reproduction in any medium, provided the original work is properly cited.
Obsessive-compulsive disorder (OCD) is a debilitating neuropsychiatric condition, characterised by repetitive intrusive thoughts and/or repetitive behaviours undertaken according to rigid rules (or in response to obsessions). ${ }^{1}$ Neurobiological models of OCD emphasise dysfunction of the basal ganglia, implicated in generating habitual behaviour. ${ }^{2,3}$ In a meta-analysis of neuroimaging studies, structural abnormalities of the basal ganglia were identified in patients with OCD versus controls, coupled with abnormal functional activation in this region during tests of executive functions, including cognitive flexibility. ${ }^{4}$ Other meta-analysis did not find macroscopic volumetric basal ganglia abnormalities in $\mathrm{OCD},{ }^{5}$ but inconsistencies may reflect the variable methodological quality of the included data studies. Although most studies focused on total volumes of whole subcortical structures, advances in imaging pipelines have made it possible to examine detailed, localised changes in the surface morphology (i.e. shape) of basal ganglia structures. ${ }^{6}$ This is a promising approach to identify familial markers of OCD, because morphological basal ganglia changes were previously found in patients with OCD and their relatives, when conventional volumetric measures were insensitive. ${ }^{6}$ Furthermore, understanding the basal ganglia is also relevant for transdiagnostic approaches, because their dysfunction is implicated in other less-studied obsessive-compulsive-related disorders. ${ }^{7}$

The basal ganglia play a prominent role in habit learning and regulation (i.e. cognitive flexibility). ${ }^{7}$ One common laboratoryassessed form of cognitive flexibility is set-shifting, which is the ability to switch attention from one important aspect of the environment to another during a task, in response to changing reinforcement contingencies. ${ }^{8}$ Set-shifting is classically measured by an extradimensional shift in such tasks, whereby participants inhibit attention away from a previously relevant aspect of the environment, onto a different aspect of the environment that was previously irrelevant. For more detailed discussion of the cognitive neuroscience of extradimensional shifting and its relationship to habit and loss of top-down flexibility, see work by Robbins ${ }^{9}$ and Chamberlain et al. ${ }^{10}$ Abnormalities in this cognitive function have been extensively implicated in the pathophysiology of OCD. ${ }^{8}$ Impaired set-shifting performance on the intradimensional/extradimensional (IDED) task has been confirmed in a meta-analysis of OCD studies with medium-to-large effect size, and has also been found to extend to clinically asymptomatic first-degree relatives of patients, thereby constituting a candidate familial marker. ${ }^{10}$

Given that basal ganglia morphological changes and impaired set-shifting have separately been implicated as candidate familial markers for OCD in prior work, this study sought to demonstrate a link between the two. We hypothesised that basal ganglia shape would relate to set-shifting performance overall; and morphological abnormalities would be found in patients with OCD and their relatives versus controls, in the identified set-shifting-related brain regions.

\section{Method}

\section{Participants}

The pooled data-set included participants from two studies, where participants underwent conventional imaging measures, reported previously $^{11,12}$ (technical details of the scans are provided in Supplementary File 1 available at https://doi.org/10.1192/bjp.2021. 45). The first sample comprised 32 pairs of unaffected first-degree relatives and OCD probands, and 32 age- and gender-matched healthy controls. ${ }^{7}$ The second sample comprised 44 patients with OCD and 43 age- and gender-matched healthy controls. ${ }^{8}$

Participants were screened for mental disorders by extended clinical interview conducted by a psychiatrist, supplemented with the Mini International Neuropsychiatric Inventory (MINI). ${ }^{13}$ OCD severity was quantified with the Yale-Brown Obsessive Compulsive Scale (Y-BOCS), ${ }^{14}$ and IQ was measured by the 
National Adult Reading Test. ${ }^{15,16}$ Patients with OCD were excluded if they had any comorbidities, including depression. Controls and first-degree relatives of patients were excluded if they had any history of mental disorders. Controls were excluded if they reported any known history of OCD in first-degree family members. History of serious head injury, substance misuse, epilepsy or magnetic resonance imaging contraindications was exclusionary across all groups.

The study was approved by the Addenbrooke's NHS Trust Local Research Ethics Committee (Cambridge, UK) and Local Research Ethical Committee at the University of Cambridge (Cambridge, UK). The research complied with relevant ethical standards including the Declaration of Helsinki. All participants provided written informed consent after reading information sheets about the study and having the opportunity to ask questions.

\section{Behavioural testing}

Before scanning, all participants undertook the computerised IDED set-shift task from the Cambridge Neuropsychological Test Automated Battery (Cambridge Cognition, Cambridge, UK; see https://www.cambridgecognition.com/cantab/). Two stimuli were displayed on-screen for each trial, and participants attempted to learn underlying rules governing which stimulus was correct, based on computerised feedback. Set-shifting performance on the task was indexed by errors on the crucial extradimensional attentional shift stage. Of the different stages of the task (i.e. learning, intradimensional set-shifting, etc.) extradimensional attentional shift stage was previously found to be selectively impaired in OCD, in meta-analysis. ${ }^{10}$ Extradimensional shifting is widely regarded as the archetypal form of set-shifting on such tasks. A more detailed task description is provided in Supplementary File 1.

\section{Data analysis}

To address the two study hypotheses, our overall a priori analytic approach was first, to identify morphological regions of basal ganglia structures related to set-shifting performance; and second, to examine whether morphology in these set-shifting-related areas was abnormal in patients with OCD and their relatives versus controls.

Following standard pre-processing pipelines (for details, see Supplementary File 1), vertex analysis was deployed to investigate the shapes of subcortical structures, using FIRST from the FMRIB Software Library (FSL). ${ }^{17}$ The vertex locations of each participant were projected onto the surface normal of the average shape template of the 336 training subjects provided by FSL, and the perpendicular distance from the average surface was calculated. A positive value of the vertex indicated deformation in the outward direction, and a negative value of a vertex indicated deformation in the inward direction. 'Randomise', a permutation-based, nonparametric testing method implemented in FSL (5000 iterations), was used to identify subcortical structures whose shape was significantly associated (i.e. correlated with) with extradimensional errors, across all participants. ${ }^{18}$ Based on extant models of OCD and structures implicated in flexibility, we focused on the caudate, putamen, globus pallidus and nucleus accumbens. ${ }^{2,3}$ We performed multiple comparison correction at $P<0.05$, using threshold-free cluster enhancement.

Group differences in demographic data were explored by ANOVA or chi-squared test, as appropriate. Group differences in subcortical clusters associated with extradimensional performance were explored by non-parametric Kruskal-Wallis test, to identify overall group differences; and Wilcoxon test, for pairwise comparison of groups if overall effects of groups were found. Correlations (Spearman's rho) were examined between patient-relative pairs in terms of curvature in the identified clusters, and between randomly permuted pairs of participants, to identify whether the extradimensional-related structural changes were significantly familial. Statistical significance was defined as $P<0.05$ (uncorrected).

\section{Results}

The groups did not differ significantly from each other in terms of age, gender and estimated verbal IQ (all $P>0.10$; Supplementary Table 1). A total of 51 (67.1\%) patients in the OCD group were receiving psychotropic medication. The mean total Y-BOCS score in the OCD group was 21.89 (s.d. 5.30).

Two significant brain clusters were identified in which curvature was significantly associated with the number of extradimensional errors on the IDED task across all study participants (Fig. 1, a and b, left panel). One cluster (overall $P=0.0380$ ) was in the left putamen, maximal at $[X=-32, Y=-8, Z=-3]$ and of extent 363 voxels. The other cluster (overall $P=0.0252$ ) was in the left pallidum, maximal at $[X=-21, Y=-15, Z=-2]$ and of extent 216 voxels. The scanner type did not significantly affect curvature in these clusters (each $P>0.50$ by Wilcoxon test, comparing cluster parameters between sites).

Groups differed significantly in curvature in the left putamen cluster (Kruskal-Wallis test, $10.001 ; P=0.007$ ), but not significantly in the left pallidum cluster (Kruskal-Wallis test, 2.308; $P=0.315$ ). As shown in Fig. $1 \mathrm{a}$ and $\mathrm{b}$ (right panel), patients with OCD had significant inward curvature deformation in the left putamen cluster versus controls $(P=0.008)$, as did their relatives $(P=0.008)$. The group difference in the left putamen cluster was also significant in each study data-set considered separately: study $1, P=0.0270$; study $2, P=0.0353$. Patients did not differ significantly from relatives on this measure $(P=0.3661)$.

Mean curvature in the extradimensional-associated putamen cluster was significantly correlated in OCD proband-relative pairs $\left(r^{2}=0.261, P<0.001\right)$, but not randomly permuted pairs $(P>0.10$; bootstrap $N=2500,95 \%$ confidence interval for the null model $P$-value was $0.37-0.39$ ).

Patients with OCD who were receiving psychotropic medication did not differ from patients who were not receiving such medication, in terms of curvature in the putamen cluster (Wilcoxon test, $P=0.78$ ). Curvature in the putamen cluster did not significantly correlate with Y-BOCS total scores in patients with OCD $(P=0.233)$.

As expected, and previously reported, patients with OCD and their relatives were selectively impaired on the extradimensional stage of the IDED task versus controls (Supplementary File 1).

\section{Discussion}

By conducting an analysis of cognitive function and basal ganglia morphology from magnetic resonance imaging data, this study showed that set-shifting performance (extradimensional shifting) correlated with putamen morphology; and demonstrated that patients with OCD and their asymptomatic first-degree relatives had common abnormalities in this set-shifting-related region. Furthermore, the association between the structure of the putamen and the extradimensional errors was found to be significantly familial in OCD proband-relative pairs. These structural brain changes in patients with OCD and their relatives reflect localised (at the millimetre level) changes in the shape of specific basal ganglia structures. 
(a)
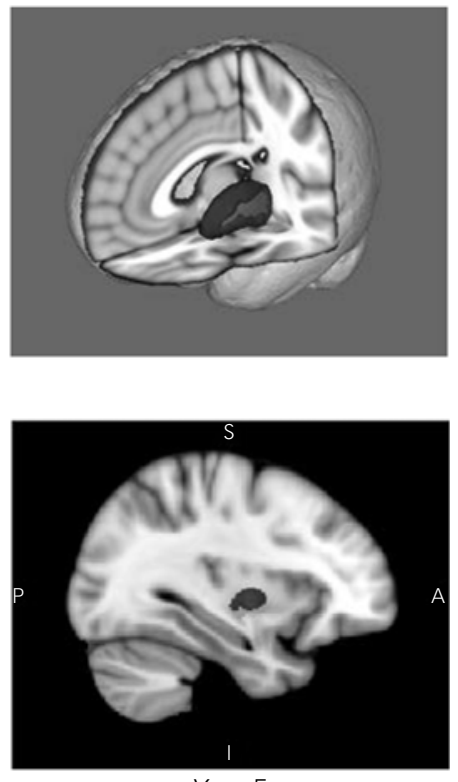

$Y=-5$

(b)
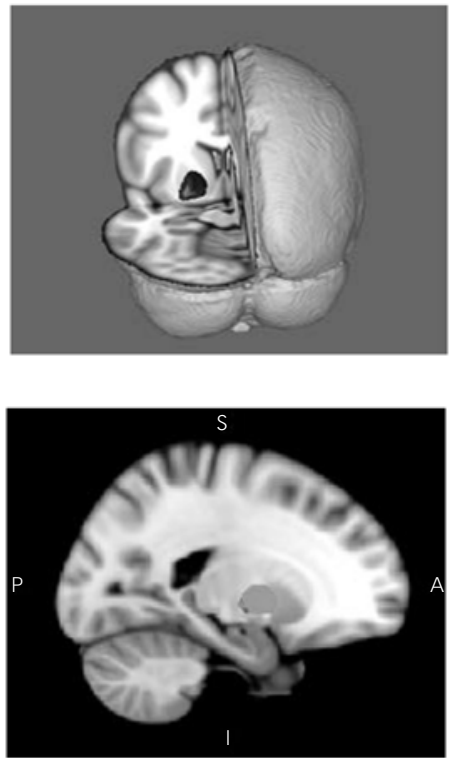

$Y=-14$

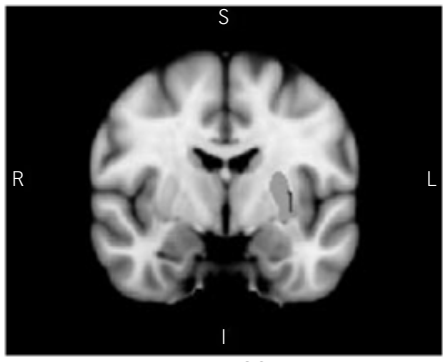

$X=-32$

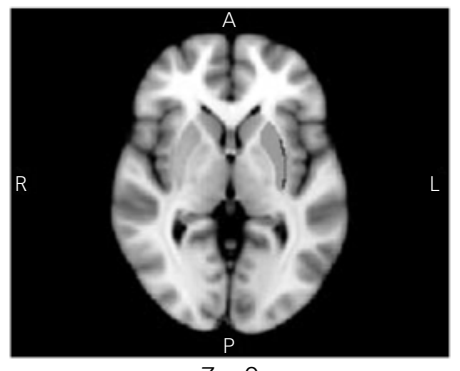

$Z=0$
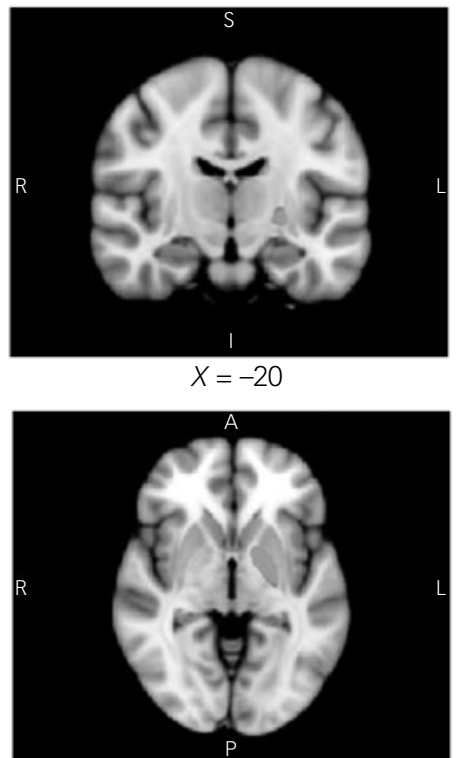

$Z=-3$
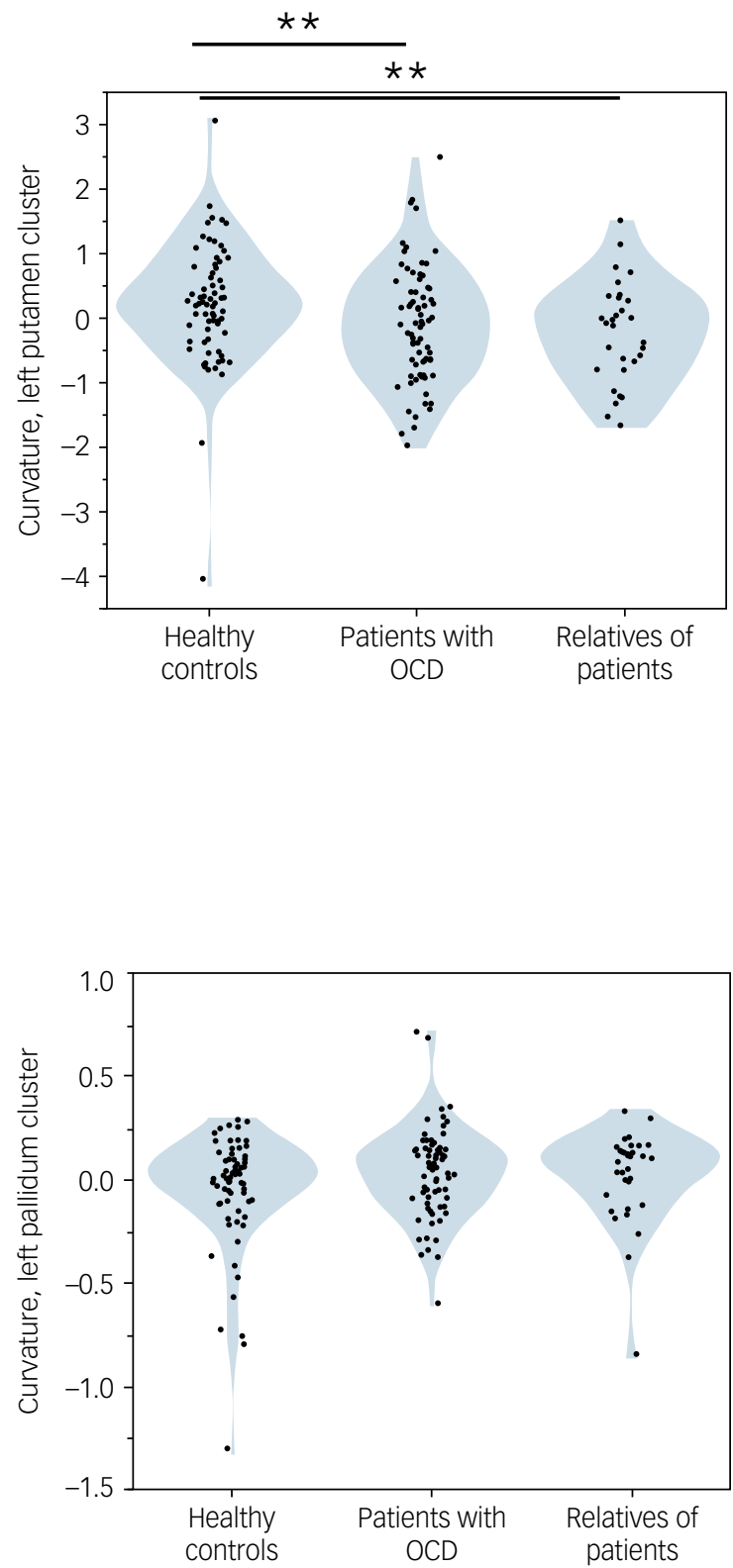

Fig. 1 (a and b) Left panel: magnetic resonance imaging of basal ganglia regions in which structural curvature was significantly associated with extradimensional shift performance. Blue indicates regions in which extradimensional errors were associated with greater inward curvature; red indicates regions in which extradimensional errors were associated with greater outward curvature. Green indicates the remainder of the given structure. ( $a$ and b) Right panel: violin plots showing curvature distributions, in each study group, for the clusters significantly associated with extradimensional performance. ${ }^{*} P<0.01$ significant difference between groups, by Wilcoxon test. Only group differences in the left putamen cluster were significant. OCD, obsessive-compulsive disorder.

The implication of these findings is that putamen shape relates to set-shifting performance, and that deformation of this structure constitutes a related candidate familial marker for OCD. The findings may reflect clustering of different groups in separate areas of the bidimensional (i.e. extradimensional shift versus brain structure) variable space. Previous translational research indicate that the putamen plays a cardinal role in learning, habitual behaviour and adapting stimulus-response associations. ${ }^{3,19}$ Extradimensional errors in OCD were previously found to be associated with reduced frontal connectivity between the dorsal striatum and frontal cortical regions. ${ }^{12}$ Thus, predilection toward habitual response patterns and impaired goal-directed control, linked to morphological changes of the basal ganglia, appear to confer familial risk for OCD. The morphological changes do not appear to be diagnostic markers or a consequence of symptoms themselves, and are not secondary to psychotropic medication. Structural abnormalities of the basal ganglia may reflect altered neurodevelopmental trajectories 
associated with the risk of OCD, but to address this would require longitudinal research.

Masanori Isobe, Department of Psychiatry, Kyoto University, Japan; Department of Psychiatry, University of Cambridge, UK; Cambridge and Peterborough NHS Foundation Trust, UK; and The Nippon Foundation, Japan; Matilde Vaghi, Department of Psychology, University of Cambridge, UK· University College London, UK. Naomi A. Fineberg, University of Hertfordshire, UK; and Hertfordshire Partnership University NHS Trust, UK; Annemieke M. Apergis-Schoute, Department of Neuroscience, Psychology and Behaviour, University of Leicester, UK; Edward T. Bullmore, Department of Psychiatry, University of Cambridge, UK; and Cambridge and Peterborough NHS Foundation Trust, UK; Barbara J. Sahakian, Department of Psychiatry, University of Cambridge, UK; Cambridge and Peterborough NHS Foundation Trust, UK; and Department of Psychology, University of Cambridge, UK; Trevor W. Robbins, Department of Psychology, University of Cambridge, UK: Samue R. Chamberlain (D), Department of Psychiatry, University of Cambridge, UK; Cambridge and Peterborough NHS Foundation Trust, UK; Department of Psychiatry, University of Southampton, UK; and Southern Health NHS Foundation Trust, UK

Correspondence: Samuel R. Chamberlain. Email: src33@cam.ac.uk

First received 17 Aug 2020, final revision 18 Jan 2021, accepted 11 Mar 2021

\section{Supplementary material}

To view supplementary material for this article, please visit https://doi.org/10.1192/bjp.2021.45.

\section{Data availability}

The data are not publicly available as participants did not consent to that.

\section{Acknowledgements}

The authors wish to thank all study participants.

\section{Author contributions}

All authors contributed to the study design, intellectual content, drafting of the manuscript, interpretation of data, and approved the manuscript for submission.

\section{Funding}

This work was funded by a Wellcome Trust clinical fellowship to S.R.C. (UK; reference numbers 110049/Z/15/Z and 110049/Z/15/A). M.I.'s role in this project was funded by a grant-in-aid for scientific research on innovative areas (grant number 16K21720) from the Ministry of Education, Culture, Sports, Science and Technology of Japan, and by the Nippon Foundation international fellowship. T.W.R., A.M.A.S. and M.V. are supported by Wellcome Trust grant 104631/Z/14/Z.

\section{Declaration of interest}

S.R.C. consults for Promentis, and receives stipends from Elsevier for journal editorial work B.J.S. and T.W.R. consult for, and receive royalties from, Cambridge Cognition. N.A.F. declares that in the past 3 years, she has held research or networking grants from the ECNP, UK NIHR, EU H2020 (COST), MRC and University of Hertfordshire; accepted travel and/or hospitality expenses from the BAP, ECNP, Royal College of Psychiatrists, CINP, International Forum of Mood and Anxiety Disorders, World Psychiatric Association, Indian Association for Biological Psychiatry, Sun; received payment from Taylor and Francis and Elsevier for editorial duties; and accepted a paid speaking engagement in a webinar sponsored by Abbott. Previously, N.A.F. has accepted paid speaking engagements in various industry-supported symposia, and recruited patients for various industry-sponsored studies in the field of OCD treatment. N.A.F. leads an NHS treatment service for OCD; holds Board membership (or similar) for various registered charities linked to OCD; and gives advice on psychopharmacology to the UK MHRA. E.T.B. is a National Institute of Health Research Senior Investigator, is full-time employed by the University of Cambridge and was previously (until May 2019) part-time employed by GlaxoSmithKline; is a member of the Scientific Advisory Board of Seiso
Heptares; and receives research funding from Janssen, GlaxoSmithKline and Lundbeck as part of the Wellcome Trust Consortium for the Neuroimmunology of Mood Disorders and Alzheimer's Disease. The other authors report no potential conflicts of interest.

\section{References}

1 American Psychiatric Association. Diagnostic and Statistical Manual of Mental Disorders, Fifth Edition. American Psychiatric Publishing, 2013.

2 Fineberg NA, Apergis-Schoute AM, Vaghi MM, Banca P, Gillan CM, Voon V et al. Mapping compulsivity in the DSM-5 obsessive compulsive and related disorders: cognitive domains, neural circuitry, and treatment. Int $J$ Neuropsychopharmacol 2018; 21(1): 42-58.

3 Graybiel AM, Rauch SL. Toward a neurobiology of obsessive-compulsive disorder. Neuron 2000; 28(2): 343-7.

4 Pico-Perez M, Moreira PS, de Melo Ferreira V, Radua J, Mataix-Cols D, Sousa N, et al. Modality-specific overlaps in brain structure and function in obsessivecompulsive disorder: multimodal meta-analysis of case-control MRI studies. Neurosci Biobehav Rev 2020; 112: 83-94.

5 Boedhoe PS, Schmaal L, Abe Y, Ameis SH, Arnold PD, Batistuzzo MC, et al. Distinct subcortical volume alterations in pediatric and adult OCD: a worldwide meta- and mega-analysis. Am J Psychiatry 2017; 174(1): 60-9.

6 Shaw P, Sharp W, Sudre G, Wharton A, Greenstein D, Raznahan A, et al. Subcortical and cortical morphological anomalies as an endophenotype in obsessive-compulsive disorder. Mol Psychiatry 2015; 20(2): 224-31.

7 Macpherson T, Hikida T. Role of basal ganglia neurocircuitry in the pathology of psychiatric disorders. Psychiatry Clin Neurosci 2019; 73(6): 289-301.

8 Chamberlain SR, Blackwell AD, Fineberg NA, Robbins TW, Sahakian BJ. The neuropsychology of obsessive compulsive disorder: the importance of failures in cognitive and behavioural inhibition as candidate endophenotypic markers. Neurosci Biobehav Rev 2005; 29(3): 399-419.

9 Robbins TW. Shifting and stopping: fronto-striatal substrates, neurochemical modulation and clinical implications. Philos Trans R Soc Lond B Biol Sci 2007; 362(1481): 917-32.

10 Chamberlain SR, Solly J, Hook R, Vaghi MM, Robbins TW. Cognitive Inflexibility in OCD and Related Disorders. Curr Top Behav Neurosci [Epub ahead of print] 6 Feb 2021. Available from: https://doi.org/10.1007/7854_2020_198.

11 Menzies L, Achard S, Chamberlain SR, Fineberg N, Chen CH, del Campo N, et al. Neurocognitive endophenotypes of obsessive-compulsive disorder. Brain 2007; 130(pt 12): 3223-36.

12 Vaghi MM, Vertes PE, Kitzbichler MG, Apergis-Schoute AM, van der Flier FE, Fineberg NA, et al. Specific frontostriatal circuits for impaired cognitive flexibility and goal-directed planning in obsessive-compulsive disorder: evidence from resting-state functional connectivity. Biol Psychiatry 2017; 81(8): 708-17.

13 Sheehan DV, Lecrubier $\mathrm{Y}$, Sheehan $\mathrm{KH}$, Amorim P, Janavs J, Weiller E, et al. The Mini-International Neuropsychiatric Interview (M.I.N.I.): the development and validation of a structured diagnostic psychiatric interview for DSM-IV and ICD-10. J Clin Psychiatry 1998; 59(Suppl 20): 22-33.

14 Goodman WK, Price LH, Rasmussen SA, Mazure C, Fleischmann RL, Hill CL, et al. The Yale-Brown Obsessive Compulsive Scale. I. Development, use, and reliability. Arch Gen Psychiatry 1989; 46(11): 1006-11.

15 Nelson HE. National Adult Reading Test. NFER-Nelson, 1982.

16 Nelson HE, Willison J. The Revised National Adult Reading Test (NART) - Test Manual. NFER-Nelson, 1991.

17 Patenaude B, Smith SM, Kennedy DN, Jenkinson M. A Bayesian model of shape and appearance for subcortical brain segmentation. Neuroimage 2011; 56(3): 907-22.

18 Winkler AM, Ridgway GR, Webster MA, Smith SM, Nichols TE. Permutation inference for the general linear model. Neuroimage 2014; 92: 381-97.

19 Jackson SAW, Horst NK, Axelsson SFA, Horiguchi N, Cockcroft GJ, Robbins TW et al. Selective role of the putamen in serial reversal learning in the marmoset. Cereb Cortex 2019; 29(1): 447-60.
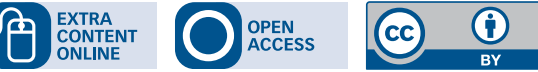